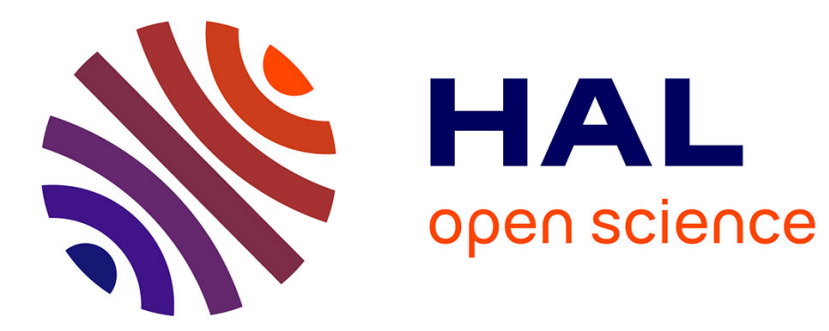

\title{
The localisation of the apical Par/Cdc42 polarity module is specifically affected in microvillus inclusion disease
}

Grégoire Michaux, Dominique Massey-Harroche, Ophélie Nicolle, Marion Rabant, Nicole Brousse, Olivier Goulet, André Le Bivic, Frank M Ruemmele

\section{- To cite this version:}

Grégoire Michaux, Dominique Massey-Harroche, Ophélie Nicolle, Marion Rabant, Nicole Brousse, et al. The localisation of the apical Par/Cdc42 polarity module is specifically affected in microvillus inclusion disease. Biology of the Cell, 2016, 108 (1), pp.19-28. 10.1111/boc.201500034 . hal-01231419

HAL Id: hal-01231419

https://hal-univ-rennes1.archives-ouvertes.fr/hal-01231419

Submitted on 25 Jan 2016

HAL is a multi-disciplinary open access archive for the deposit and dissemination of scientific research documents, whether they are published or not. The documents may come from teaching and research institutions in France or abroad, or from public or private research centers.
L'archive ouverte pluridisciplinaire HAL, est destinée au dépôt et à la diffusion de documents scientifiques de niveau recherche, publiés ou non, émanant des établissements d'enseignement et de recherche français ou étrangers, des laboratoires publics ou privés. 


\section{The localisation of the apical Par/Cdc42 polarity module is specifically affected in microvillus inclusion disease}

Grégoire Michaux 1,2,", Dominique Massey-Harroche3 ${ }^{3}$ Ophélie Nicolle 1,2, Marion Rabant ${ }^{4}$, Nicole Brousse $^{4}$, Olivier Goulet ${ }^{5,6}$, André Le Bivic ${ }^{3}$, Frank M Ruemmele,5,6,7

${ }^{1}$ CNRS, UMR6290, Institute de Génétique et Développement de Rennes, F-35043 Rennes, France

2 University de Rennes 1, UEB, SFR Biosit, Faculté de Médecine, F-35043 Rennes, France

3 Aix-Marseille Université, CNRS, UMR 7288, Developmental Biology Institute of Marseille (IBDM), case 907, 13288 Marseille cedex 09, France

${ }^{4}$ Pathology department, Hôpital Necker-Enfants Malades, Paris, France

${ }^{5}$ Pediatric Gastroenterology Unit, Hôpital Necker-Enfants Malades, AP-HP, Paris, France

${ }^{6}$ Université Paris Descartes, Sorbonne Paris Cité, Paris, France

7 Institute Imagine, INSERM U1163, Paris, France

Running title: Polarity defects in MVID

Keywords: MVID - Epithelial polarity - Membrane traffic - Myo5B - tricho-hepato-enteric syndrome - celiac disease 
* Corresponding author: Grégoire Michaux

CNRS UMR6290 - 2 avenue du Pr Léon Bernard - 35043 Rennes - France

Tel: (00 33) -2 2323 4775; email: gmichaux@univ-rennes1.fr 


\section{ABSTRACT}

\section{Background Information}

Microvillus inclusion disease (MVID) is a genetic disorder affecting intestinal absorption. It is caused by mutations in MYO5B or syntaxin 3 (STX3) affecting apical membrane trafficking. Morphologically MVID is characterised by a depletion of apical microvilli and the formation of microvillus inclusions inside the cells, suggesting a loss of polarity. To investigate this hypothesis we examined the location of essential apical polarity determinants in five MVID patients.

Results

We found that the polarity determinants Cdc42, Par6B, PKC // 1 and the structural proteins ezrin and phospho-ezrin were lost from the apical membrane and accumulated either in the cytoplasm or on the basal side of enterocytes in patients which suggests an inversion of cell polarity. Moreover microvilli-like structures were observed at the basal side in electron microscopy. We next performed Myo5B depletion in 3D-grown human Caco2 cells forming cysts and we found a direct link between the loss of Myo5B and the mislocalisation of the same apical proteins; furthermore we observed that a majority of cyst displayed an inverted polarity phenotype as seen in some patients. Finally we found that this loss of polarity was specific for MVID: tissue samples of patients with Myo5B independent absorption disorders showed normal polarity but we identified Cdc42 as a potentially essential biomarker for tricho-hepato-enteric syndrome.

\section{Conclusion}

Our findings indicate that the loss of Myo5B induces a strong loss of enterocyte polarity, potentially leading to polarity inversion. 


\section{Significance}

Our results show that polarity determinants could be useful markers to help establishing a diagnosis in patients. Furthermore they could be used to characterise other rare intestinal absorption diseases. 


\section{INTRODUCTION}

Microvillus inclusion disease (MVID, also called microvillus atrophy, OMIM 251850) is a genetic disorder characterised morphologically by a loss of apical microvilli and formation of microvillus inclusions in the cytoplasm of enterocytes. The clinical picture in patients with MVID is characterised by severe secretory diarrhea most often starting the first days of life, which can be rapidly life-threatening. MVID is caused in $90 \%$ of cases by mutations affecting myosin 5B (MYO5B) which encodes a molecular motor implicated in apical transport (Muller et al., 2008). Other patients were shown to carry mutations in the apical protein syntaxin 3 (STX3) (Wiegerinck et al., 2014). Apical accumulation of periodic acid Schiff (PAS) positive granules and the abnormal staining of the apical marker CD10 were identified early as reliable markers for MVID (Groisman et al., 2002; Phillips et al., 2000). Following these studies, many other apical markers have been identified as affected in MVID patients, such as cystic fibrosis transmembrane conductance, sucrase isomaltase, alkaline phosphatase (Ameen and Salas, 2000), ezrin, phospho-ezrin and aPKCı (Dhekne et al., 2014), villin (Muller et al., 2008; Shillingford et al., 2015) and phosphoinositide-dependent protein kinase 1 (PDK1) (Kravtsov et al., 2014). Indeed the cellular defects associated with MVID suggest a disturbed apical traffic as shown recently by several studies (Carton-Garcia et al., 2015; Dhekne et al., 2014; Knowles et al., 2014; Kravtsov et al., 2014; Schneeberger et al., 2015; Thoeni et al., 2014).

Intestinal cells are polarised with an apical membrane composed of microvilli forming the brush border and of the terminal web (Crawley et al., 2014). But like all epithelial cells, intestinal cells rely on polarity determinants to establish and maintain their polarity (Overeem et al., 2015). The apical Cdc42/Par module composed of PAR-6, aPKC, PAR-3 and Cdc42 is the most essential and universally conserved polarity module since it is required in almost all polarised cells, from asymmetrically dividing cells to neurons, as well as in migrating cells or during the formation of the immunological synapse: the association of this module to a membrane domain is enough to determine the fate of this membrane (Etienne-Manneville, 2004; Suzuki and Ohno, 2006). Several studies demonstrated that membrane trafficking is required for the localisation of the apical Par/Cdc42 polarity module (Apodaca et al., 2012) and aPKCı has been shown to be mislocalised in MVID patients (Dhekne et al., 2014). However the severity of the loss of polarity 
phenotype associated with MVID has not been investigated before. We therefore decided to systematically examine the localisation of the apical Par/Cdc42 module, as well as ezrin and phospho-ezrin, in five MVID patients and in Myo5B depleted 3D cultured Caco2 cells. We show that polarity determinants are mislocalised following the loss of Myo5B and this loss of polarity can even lead to a severe inverted polarity phenotype. 


\section{RESULTS AND DISCUSSION}

\section{The apical Par/Cdc42 polarity determinants are depleted from the apical pole and accumulate intracellularly in MVID patients}

The cellular distribution of the polarity determinants Par6B, PKC // (isoforms of aPKC) and Cdc42 were analysed in five MVID patient samples by immunohistochemistry (IHC) and compared to normal tissue samples from children of a similar age (Fig1). In normal duodenal

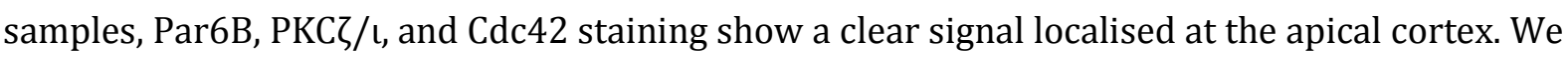
also examined the localisation of ezrin and phospho-ezrin (P-ezrin) which are essential factors for the formation of the terminal web and microvilli; P-ezrin is restricted to the most apical part of enterocytes corresponding to microvilli and ezrin is an apical cortical protein. In contrast to healthy samples, a highly pathological phenotype was observed in all patients with MVID: Four patients (Patients no 1-2-4-5, Table S1) showed an identical phenotype (Fig1A) characterised by ezrin depletion from the apical pole and accumulation inside the cell, and completely absent signal for P-ezrin (Fig1). PKCろ/ı was also depleted from the most apical parts of enterocytes. Par6B staining was negative at the apical membrane but positive inside the cell. Cdc42 staining was lost from the apical cortex in all MVID patients. Systematic quantification (see Material and Methods) confirmed the relative loss of apical localisation compared to cytoplasmic accumulation for each marker in one representative patient (Fig1B). We concluded that the abnormal localisation of apical polarity determinants demonstrates that enterocyte polarity is severely affected in these patients. We also confirmed a recent report showing that ezrin, Pezrin and PKCı were mislocalised in two MVID patients (Dhekne et al., 2014).

\section{Polarity inversion in two MVID patients}

In 3 out of 6 circular folds visible on the tissue sample from MVID patient 3 (Fig2) we observed the same staining pattern as described above (not shown). However in the other circular folds, up to half villi displayed a different staining pattern: Par6B, PKC / /, Cdc42 as well as ezrin/Pezrin were completely depleted from the apical side but were found accumulating at the level of the basal cortex (Fig2); a similar observation was made less frequently in patient 4 (Fig2). On electron microscopy (EM) analyses in patient 3 (Fig2) we did not find microvillus inclusions but 
microvilli were partially depleted from the apical pole, consistent with the microvillus atrophy systematically found in MVID, while microvilli-like structures were detected at the basal membrane. These observations suggested a complete polarity inversion; however nuclei were found at their normal position close to the basal pole of the cells and we did not observed a pseudostratified epithelium. These two phenotypes were found in patients mutated for TTC7A leading to multiple intestinal atresia (MIA) which also display polarity inversion (Bigorgne et al., 2014). We therefore concluded that MVID can induce a less severe partial polarity inversion with respect to the localisation of apical polarity determinants and of the brush border. This patient had a $M Y O 5 B$ mutation introducing a stop codon which could generate a potentially dominant-negative version of Myo5B. But because this particular phenotype was only observed in some villi, such a dominant-negative function is unlikely. To our knowledge concomitant formation of ectopic microvilli and localisation of several polarity determinants in vivo has only been observed in C. elegans (Shafaq-Zadah et al., 2012; Zhang et al., 2011; Zhang et al., 2012). These results support the hypothesis of an essential role for membrane traffic in the localisation of polarity determinants and therefore on brush border formation and maintenance in vivo.

\section{Myo5B depletion in 3D-grown Caco2 cells leads to polarity inversion}

To ask whether the defects observed in patients were directly due to Myo5B depletion or secondary defects, we used an in vitro system. To mimic enterocytic morphogenesis of the intestinal epithelium, Caco2 cells were grown in three dimensions (3D) in Matrigel to provide a more physiological growth pattern and the formation of cysts with a central lumen rather than a flat epithelium. To knock down $M Y O 5 B$, an shRNA transduction lentiviral approach was chosen which yielded an 78\% and 84\% decrease in Myo5B expression, respectively with shMyo5B-58

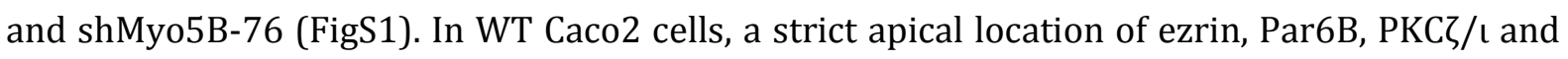
of other apical markers such as F-actin and DPPIV was observed (Fig3A-D). However upon Myo5B depletion ezrin, P-ezrin, Par6B and aPKC $/ /$ were detected on the basal side of the cells (Fig3A-D). The persistent apical localisation of all markers is likely due to the incompletely penetrant decrease in Myo5B expression. We concluded that this model system can be used to mimic the abnormal localisation of polarity determinants observed in some patients. This result is different from observations made in 2D-grown Caco2 or Caco2 $2_{\mathrm{BBE}}$ cells by several groups including ourselves (Dhekne et al., 2014; Knowles et al., 2014; Kravtsov et al., 2014; Ruemmele et al., 2010; Thoeni et al., 2014). Apart from the Myo5B depletion efficiency, one hypothesis to 
explain this discrepancy is that growing Caco2 cells as a flat epithelium or as a cyst mimicking the lumenal structure of the intestine could differentially reveal phenotypes and indeed many studies have reported differences in 2D versus 3D grown cells (Yamada and Cukierman, 2007). More recently $M Y O 5 B$ knock out mice have been generated (Carton-Garcia et al., 2015; Schneeberger et al., 2015); it was shown that basolateral markers such as Na/K ATPase and Ecadherin were found at the apical side and that basolateral polarity was disturbed (Schneeberger et al., 2015). This observation could be linked to our finding that apical polarity determinants can be lost from the apical membrane and mistargetted to the basal membrane. It will be important to confirm this hypothesis by localising Cdc42 and the Par module in MYO5B knock out mice or in organoids generated from these mice.

\section{Localisation of apical polarity determinants and ezrin/P-ezrin in other absorption disorders: celiac disease and THE syndrome}

We next addressed the question if the mislocalisation of apical polarity determinants in MVID patients is specific. We therefore compared these samples to inflammatory duodenal samples (celiac disease; OMIM 212750; Table S1) as well as an absorption disorder with marked diarrhea and a disorganised intestinal epithelial structure (tricho-hepato-enteric (THE) syndrome, also called syndromic diarrhea; OMIM 222470; Table S1). Celiac disease is associated with $H L A-D Q 2 / D Q 8$ in most cases (Sollid, 2002), and THE syndrome is caused by mutations affecting TTC37 or SKIV2L, two genes implicated in RNA metabolism (Fabre et al., 2012; Hartley et al., 2010). In inflammatory tissue samples of two celiac disease patients, we did not detect any change in the location of any polarity determinants studied (Fig4A). On immunohistochemical analyses in duodenal tissues of children with THE syndrome the staining signals for Par6B, PKC $/ \mathrm{l}$, ezrin and P-ezrin were normal at the apical membrane. In contrast a consistent and specific loss of Cdc42 was observed (Fig4A) which affected all intestinal cell types in samples of all three THE syndrome patients; this phenotype was quantified (Fig4B) and confirmed with two different antibodies (FigS2).

These data suggest that we identified a potential biomarker, Cdc42, for THE syndrome. In contrast to MVID, THE syndrome has a wide clinical spectrum beyond the small intestine such as woolly hair, facial dysmorphism and immunodeficiency (Fabre et al., 2013). Why would 
Cdc42 be specifically lost in these patients where RNA metabolism is perturbed is not clear at that stage but it provides an interesting hypothesis to explain villous atrophy: Cdc42 is essential for the correct orientation of cell division in 3D grown Caco2 cells and for the small intestine morphogenesis in mouse (Jaffe et al., 2008; Melendez et al., 2013; Sakamori et al., 2012) and could therefore control villi morphogenesis in humans. Future work could explore this lead by characterising Cdc42 expression in other affected tissues. It is also surprising to find that Cdc42 can be missing from the apical membrane without affecting the rest of the apical Par module nor the localisation of ezrin and P-ezrin, indicating that $\mathrm{Cdc} 42$ is not absolutely essential at least for polarity maintenance in enterocytes.

It has been suggested that several other rare genetic absorption diseases could be linked either to polarity inversion (Bigorgne et al., 2014) or disrupted apical microvilli (Salomon et al., 2014; Stepensky et al., 2013). Our results obtained in MVID and THE syndrome suggest that localising the apical Par/Cdc42 module could yield clinically relevant results to analyse the severity of polarity defects associated with intestinal absorption disorders. Contrary to markers or methods often used to characterise the apical membrane of enterocytes, such as PAS staining or CD10, localising the apical Par/Cdc42 module brings another level of information which is due to the function of these factors in directly establishing and maintaining epithelial polarity. 


\section{MATERIALS AND METHODS}

\section{Patient Material}

Small bowel biopsies (duodenum) or resections from patients with a molecular or clinical diagnosis of MVID (mutation in $M Y O 5 B, \mathrm{n}=4$; mutation unknown, $\mathrm{n}=1$; Table S1) or clinical diagnosis of Tricho-Hepato-Enteric THE Syndrome ( $n=3$; Table S1) were used for this study. Control samples are derived from normal tissue (n=3; Table S1) or patients with celiac disease $(n=2$; Table S1) of the Necker tissue bank. This study is part of a larger project that was approved by our local ethics committee (CPP 2011-01-01 for the AMVILLO project).

\section{Antibodies}

We used anti-Par6B (PARD6B, sc-67393 Santa Cruz, 1/100), anti-Cdc42 (CDC42, ab64533,

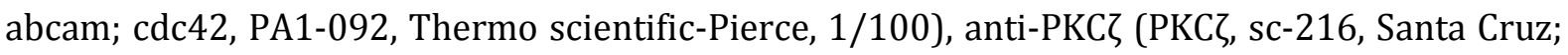
referred to as $\mathrm{PKC} / \mathrm{/}$ because it also recognises PKCı, 1/200), anti-ezrin (Ezrin C-19, sc-6407, Santa Cruz, 1/200), anti-P-ezrin (Anti-Ezrin (phospho T567), ab47293, abcam, 1/1000), antiMyosin 5B (provided by L.A. Huber, Medical University Innsbruck, Austria), Phalloidin TexasRed (Sigma aldrich) and rat monoclonal anti-DiPeptidyl Peptidase (DPPIV, for details see (Gorvel et al., 1991).

\section{Immunohistochemistry (IHC), quantifications and electron microscopy (EM)}

Paraffin-embedded tissue was cut at $4 \mu \mathrm{m}$, mounted on positively charged slides and dried at $58^{\circ} \mathrm{C}$ for 60 minutes. Immunohistochemical staining was performed on the Discovery Automated IHC stainer using the Ventana DABMap and OMNIMap detection kit (Ventana Medical Systems, Tucson, Ariz). Antigen retrieval was performed using Ventana proprietary, Tris-based buffer solution $\mathrm{CC} 1$ at $95^{\circ} \mathrm{C}$ to $100^{\circ} \mathrm{C}$ for 48 minutes. Endogen peroxidase was blocked with Inhibitor-D 3\% H2O2 (Ventana) for 8 minutes at $37^{\circ} \mathrm{C}$. After rinsing, slides were incubated at $37^{\circ} \mathrm{C}$ for 60 minutes with primary antibodies. Signal enhancement was performed using the Ventana DABMap Kit for PARD6B, PKC $/$ / , P-ezrin or Ventana ChromoMap kit for 
Ezrin and Cdc42. Slides were then counterstained for 16 minutes with hematoxylin and rinsed. After removal from the instrument, slides were manually dehydrated and coverslipped.

The quantification method used in Fig1 and Fig4 is adapted from (Gillard et al., 2015; ShafaqZadah et al., 2012). Using ImageJ 1.43 we quantified the signal along straight lines about $40 \mu \mathrm{m}$ long and $2.3 \mu \mathrm{m}$ large over the apical membrane and the cytoplasm (close to the nuclei); the line spanned about 5 cells on average (red line in Fig1). The apical signal was normalised to the cytoplasmic signal to measure the relative apical or cytoplasmic enrichment. A ratio of 1 therefore indicates no specific cortical staining; above 1 indicates an apical enrichment and below 1 indicates a cytoplasmic enrichment. Similar measures were performed in 10 different villi for controls and patients.

Electron microscopy was performed following standard procedures. Thin sections on grids were viewed using a JEM-1400 transmission electron microscope (JEOL, Tokyo, Japan) equipped with a Gatan Orius SC 1000 camera.

\section{Myosin 5B knockdown, Caco2 3D cell culture and immunofluorescence}

A Caco2 clone (TC7 cells) was grown as described previously (Chantret et al., 1994; Vacca et al., 2014) and used to knockdown Myosin 5B by RNA interference with Mission shRNA Lentiviral Transduction Particles (SHCLNV-NM_001080467: clone TRCN0000265458 and TRCN0000254076, called respectively shMyo5B-58 and shMyo5B-76, Sigma-Aldrich) according to the manufacturer's instructions. Transduction with Lentiviral Transduction Particles containing an empty vector (SHC002v, called shMyo5B-Ct, Sigma-Aldrich) were used as control. After $18 \mathrm{~h}$ post infection, shRNA-containing cells were selected with $12 \mathrm{~g} / \mathrm{ml}$ puromycin and Myo5B expression was monitored by western blot analysis as described (Lemmers et al., 2002) (Fig S2). To produce cysts, cells were trypsinized and resuspended in media containing 4\% Matrigel (BD Biosciences) and $10^{5}$ cells of this suspension was plated on Matrigel precoated coverslips in a 4-well plate ( $\mathrm{Nunc}^{\mathrm{TM}}$, Thermo scientific). Three-dimensional cysts were grown on top of Matrigel and maintained for 8-10 days in culture. The medium with 4\% matrigel was changed every 4 days. Although cholera toxin can be added to induce the expansion of a large 
central lumen (Jaffe et al., 2008) we decided not to use it to prevent any effect on polarity potentially induced by the toxin binding to lipid rafts (Danielsen and Hansen, 2006).

Caco2 cysts on coverslips were fixed in 3\% paraformaldehyde/PBS for 15 minutes, permeabilized in $0.5 \%$ Triton X-100/PBS (phosphate buffered saline) for 10 minutes then treated with glycine $100 \mathrm{mM} / \mathrm{PBS}$ for 20 minutes and then incubated in $10 \%$ calf fetal serum/PBS for 1 hour. Next, Caco2 cysts were incubated in 10\% calf fetal serum/PBS, $0.2 \%$ Triton X-100, 0.05\% Tween 20 supplemented with primary antibodies overnight at $4^{\circ} \mathrm{C}$ and washed extensively. Then coverslips were incubated with the appropriate fluorochromeconjugated secondary antibodies during one hour at room temperature and again rinsed extensively with PBS. Finally, cysts on coverslips were mounted using ProLong antifade reagent (Molecular Probes) and visualized using an inverted ZEISS LSM510 Meta confocal microscope (Zeiss, Le Pecq, France). The quantification of cysts with central or partial-inverted (basal) location of polarity determinants were performed in three independent experiments and in addition the cysts were classified according to lumen number into two major classes with single or multiple lumens. Per condition $>100$ cysts/experiment were analyzed and standard deviation calculated. 


\section{ACKNOWLEDGMENTS}

We are very grateful to Anne Pacquelet and Geneviève de Saint Basile for critical reading of the manuscript and to Nadine Cerf Bensussan for her support. We are deeply indebted to Pascale Bellaud and Alain Fautrel from the H2P2 histopathology facility (Rennes, France); we also thank the photonic and electron microscopy facilities of the Microscopy Rennes Imaging Center (MRic, Rennes, France) and France-BioImaging infrastructure supported by the Agence Nationale de la Recherche (ANR-10-INSB-04-01, call "Grand Emprunt"). The authors declare no conflict of interest.

\section{AUTHOR CONTRIBUTIONS}

G.M., A.L.B. and F.M.R conceived and designed the experiments. G.M., D.M.H. and O.N. performed the experiments. M.R., N.B. and O.G. provided tissue patients. G.M., D.M.H., A.L.B. and F.M.R. analysed the data and wrote the paper.

\section{FUNDING}

This work was supported mostly by an Aviesan - ITMO BCDE grant to GM and FMR; the group of GM is also supported by the Ligue contre le Cancer - 22/29/35/72, the Fondation Maladies Rares, the CNRS and Universite de Rennes 1. DMH and ALB are supported by INSERM (EOTP 404111/2), the CNRS (UMR7288) and Aix-Marseille University. The Le Bivic group is an "Equipe labellisée 2008 de la Ligue Nationale contre le Cancer". 


\section{BIBLIOGRAPHY}

Ameen, N. A. and Salas, P. J. (2000) Microvillus inclusion disease: a genetic defect affecting apical membrane protein traffic in intestinal epithelium Traffic 1, 76-83.

Apodaca, G., Gallo, L. I. and Bryant, D. M. (2012) Role of membrane traffic in the generation of epithelial cell asymmetry Nat Cell Biol 14, 1235-1243.

Bigorgne, A. E., Farin, H. F., Lemoine, R., Mahlaoui, N., Lambert, N., Gil, M., Schulz, A., Philippet, P., Schlesser, P., Abrahamsen, T. G., et al. (2014) TTC7A mutations disrupt intestinal epithelial apicobasal polarity The Journal of clinical investigation 124, 328-337.

Carton-Garcia, F., Overeem, A. W., Nieto, R., Bazzocco, S., Dopeso, H., Macaya, I., Bilic, J., Landolfi, S., Hernandez-Losa, J., Schwartz, S., Jr., et al. (2015) Myo5b knockout mice as a model of microvillus inclusion disease Sci Rep 5, 12312.

Chantret, I., Rodolosse, A., Barbat, A., Dussaulx, E., Brot-Laroche, E., Zweibaum, A. and Rousset, M. (1994) Differential expression of sucrase-isomaltase in clones isolated from early and late passages of the cell line Caco-2: evidence for glucose-dependent negative regulation J Cell Sci 107 ( Pt 1), 213-225.

Crawley, S. W., Mooseker, M. S. and Tyska, M. J. (2014) Shaping the intestinal brush border The Journal of cell biology 207, 441-451.

Danielsen, E. M. and Hansen, G. H. (2006) Lipid raft organization and function in brush borders of epithelial cells Molecular membrane biology 23, 71-79.

Dhekne, H. S., Hsiao, N. H., Roelofs, P., Kumari, M., Slim, C. L., Rings, E. H. and van Ijzendoorn, S. C. (2014) Myosin Vb and Rab11a regulate phosphorylation of ezrin in enterocytes J Cell Sci 127, 1007-1017.

Etienne-Manneville, S. (2004) Cdc42--the centre of polarity J Cell Sci 117, 1291-1300.

Fabre, A., Charroux, B., Martinez-Vinson, C., Roquelaure, B., Odul, E., Sayar, E., Smith, H., Colomb, V., Andre, N., Hugot, J. P., et al. (2012) SKIV2L mutations cause syndromic diarrhea, or trichohepatoenteric syndrome American journal of human genetics 90, 689-692.

Fabre, A., Martinez-Vinson, C., Goulet, O. and Badens, C. (2013) Syndromic diarrhea/Trichohepato-enteric syndrome Orphanet journal of rare diseases $\mathbf{8}, 5$.

Gillard, G., Shafaq-Zadah, M., Nicolle, O., Damaj, R., Pecreaux, J. and Michaux, G. (2015) Control of E-cadherin apical localisation and morphogenesis by a SOAP-1/AP-1/clathrin pathway in C. elegans epidermal cells Development 142, 1672-1683. 
Gorvel, J. P., Ferrero, A., Chambraud, L., Rigal, A., Bonicel, J. and Maroux, S. (1991) Expression of sucrase-isomaltase and dipeptidylpeptidase IV in human small intestine and colon Gastroenterology 101, 618-625.

Groisman, G. M., Amar, M. and Livne, E. (2002) CD10: a valuable tool for the light microscopic diagnosis of microvillous inclusion disease (familial microvillous atrophy) The American journal of surgical pathology 26, 902-907.

Hartley, J. L., Zachos, N. C., Dawood, B., Donowitz, M., Forman, J., Pollitt, R. J., Morgan, N. V., Tee, L., Gissen, P., Kahr, W. H., et al. (2010) Mutations in TTC37 cause trichohepatoenteric syndrome (phenotypic diarrhea of infancy) Gastroenterology 138, 2388-2398, 2398 e2381-2382.

Jaffe, A. B., Kaji, N., Durgan, J. and Hall, A. (2008) Cdc42 controls spindle orientation to position the apical surface during epithelial morphogenesis The Journal of cell biology 183, 625633.

Knowles, B. C., Roland, J. T., Krishnan, M., Tyska, M. J., Lapierre, L. A., Dickman, P. S., Goldenring, J. R. and Shub, M. D. (2014) Myosin Vb uncoupling from RAB8A and RAB11A elicits microvillus inclusion disease The Journal of clinical investigation 124, 2947-2962.

Kravtsov, D., Mashukova, A., Forteza, R., Rodriguez, M. M., Ameen, N. A. and Salas, P. J. (2014) Myosin $5 \mathrm{~b}$ loss of function leads to defects in polarized signaling: implication for microvillus inclusion disease pathogenesis and treatment American journal of physiology. Gastrointestinal and liver physiology 307, G992-G1001.

Lemmers, C., Medina, E., Delgrossi, M. H., Michel, D., Arsanto, J. P. and Le Bivic, A. (2002) hINADl/PATJ, a homolog of discs lost, interacts with crumbs and localizes to tight junctions in human epithelial cells The Journal of biological chemistry 277, 2540825415.

Melendez, J., Liu, M., Sampson, L., Akunuru, S., Han, X., Vallance, J., Witte, D., Shroyer, N. and Zheng, Y. (2013) Cdc42 coordinates proliferation, polarity, migration, and differentiation of small intestinal epithelial cells in mice Gastroenterology 145, 808-819.

Muller, T., Hess, M. W., Schiefermeier, N., Pfaller, K., Ebner, H. L., Heinz-Erian, P., Ponstingl, H., Partsch, J., Rollinghoff, B., Kohler, H., et al. (2008) MYO5B mutations cause microvillus inclusion disease and disrupt epithelial cell polarity Nature genetics 40, 1163-1165.

Overeem, A. W., Bryant, D. M. and van, I. S. C. (2015) Mechanisms of apical-basal axis orientation and epithelial lumen positioning Trends Cell Biol 25, 476-485.

Phillips, A. D., Szafranski, M., Man, L. Y. and Wall, W. J. (2000) Periodic acid-Schiff staining abnormality in microvillous atrophy: photometric and ultrastructural studies Journal of pediatric gastroenterology and nutrition 30, 34-42. 
Ruemmele, F. M., Muller, T., Schiefermeier, N., Ebner, H. L., Lechner, S., Pfaller, K., Thoni, C. E., Goulet, O., Lacaille, F., Schmitz, J., et al. (2010) Loss-of-function of MYO5B is the main cause of microvillus inclusion disease: 15 novel mutations and a CaCo-2 RNAi cell model Human mutation 31, 544-551.

Sakamori, R., Das, S., Yu, S., Feng, S., Stypulkowski, E., Guan, Y., Douard, V., Tang, W., Ferraris, R. P., Harada, A., et al. (2012) Cdc42 and Rab8a are critical for intestinal stem cell division, survival, and differentiation in mice The Journal of clinical investigation 122, 10521065.

Salomon, J., Goulet, O., Canioni, D., Brousse, N., Lemale, J., Tounian, P., Coulomb, A., Marinier, E., Hugot, J. P., Ruemmele, F., et al. (2014) Genetic characterization of congenital tufting enteropathy: epcam associated phenotype and involvement of SPINT2 in the syndromic form Human genetics 133, 299-310.

Schneeberger, K., Vogel, G. F., Teunissen, H., van Ommen, D. D., Begthel, H., El Bouazzaoui, L., van Vugt, A. H., Beekman, J. M., Klumperman, J., Muller, T., et al. (2015) An inducible mouse model for microvillus inclusion disease reveals a role for myosin $\mathrm{Vb}$ in apical and basolateral trafficking Proceedings of the National Academy of Sciences of the United States of America

Shafaq-Zadah, M., Brocard, L., Solari, F. and Michaux, G. (2012) AP-1 is required for the maintenance of apico-basal polarity in the C. elegans intestine Development 139, 20612070.

Shillingford, N. M., Calicchio, M. L., Teot, L. A., Boyd, T., Kurek, K. C., Goldsmith, J. D., Bousvaros, A., Perez-Atayde, A. R. and Kozakewich, H. P. (2015) Villin immunohistochemistry is a reliable method for diagnosing microvillus inclusion disease The American journal of surgical pathology 39, 245-250.

Sollid, L. M. (2002) Coeliac disease: dissecting a complex inflammatory disorder Nature reviews. Immunology 2, 647-655.

Stepensky, P., Bartram, J., Barth, T. F., Lehmberg, K., Walther, P., Amann, K., Philips, A. D., Beringer, O., Zur Stadt, U., Schulz, A., et al. (2013) Persistent defective membrane trafficking in epithelial cells of patients with familial hemophagocytic lymphohistiocytosis type 5 due to STXBP2/MUNC18-2 mutations Pediatric blood \& cancer 60, 1215-1222.

Suzuki, A. and Ohno, S. (2006) The PAR-aPKC system: lessons in polarity J Cell Sci 119, 979-987.

Thoeni, C. E., Vogel, G. F., Tancevski, I., Geley, S., Lechner, S., Pfaller, K., Hess, M. W., Muller, T., Janecke, A. R., Avitzur, Y., et al. (2014) Microvillus inclusion disease: loss of Myosin vb disrupts intracellular traffic and cell polarity Traffic 15, 22-42. 
Vacca, B., Bazellieres, E., Nouar, R., Harada, A., Massey-Harroche, D. and Le Bivic, A. (2014) Drebrin E depletion in human intestinal epithelial cells mimics Rab8a loss of function Human molecular genetics 23, 2834-2846.

Wiegerinck, C. L., Janecke, A. R., Schneeberger, K., Vogel, G. F., van Haaften-Visser, D. Y., Escher, J. C., Adam, R., Thoni, C. E., Pfaller, K., Jordan, A. J., et al. (2014) Loss of syntaxin 3 causes variant microvillus inclusion disease Gastroenterology 147, 65-68 e10.

Yamada, K. M. and Cukierman, E. (2007) Modeling tissue morphogenesis and cancer in 3D Cell 130, 601-610.

Zhang, H., Abraham, N., Khan, L. A., Hall, D. H., Fleming, J. T. and Gobel, V. (2011) Apicobasal domain identities of expanding tubular membranes depend on glycosphingolipid biosynthesis Nat Cell Biol 13, 1189-1201.

Zhang, H., Kim, A., Abraham, N., Khan, L. A., Hall, D. H., Fleming, J. T. and Gobel, V. (2012) Clathrin and AP-1 regulate apical polarity and lumen formation during C. elegans tubulogenesis Development 139, 2071-2083. 


\section{FIGURE LEGENDS}

\section{Figure 1: Loss of apical localisation of polarity determinants in MVID patients}

A) Ezrin, P-ezrin, Par6B, Cdc42 and PKCろ/ı staining in control and MVID enterocytes. All these markers are concentrated at the apical membrane (arrows) in control enterocytes (left panels) but are depleted from the apical membrane and accumulate intracellularly (arrowheads) except P-ezrin which is lost in MVID enterocytes (two typical patients, middle and right panels).

B) Quantification of the apical/cytoplasmic ratio for each marker in control 1 and MVID patient 1. The signal was measured along a straight line as shown for $\mathrm{PKC \zeta /ı} \mathrm{staining} \mathrm{(red} \mathrm{lines).} \mathrm{Similar}$ measures were performed in ten different villi and the average ratio is shown. Ratios $<1$ indicate a cytoplasmic enrichment relative to apical localisation. Error bars are S.E.M. 


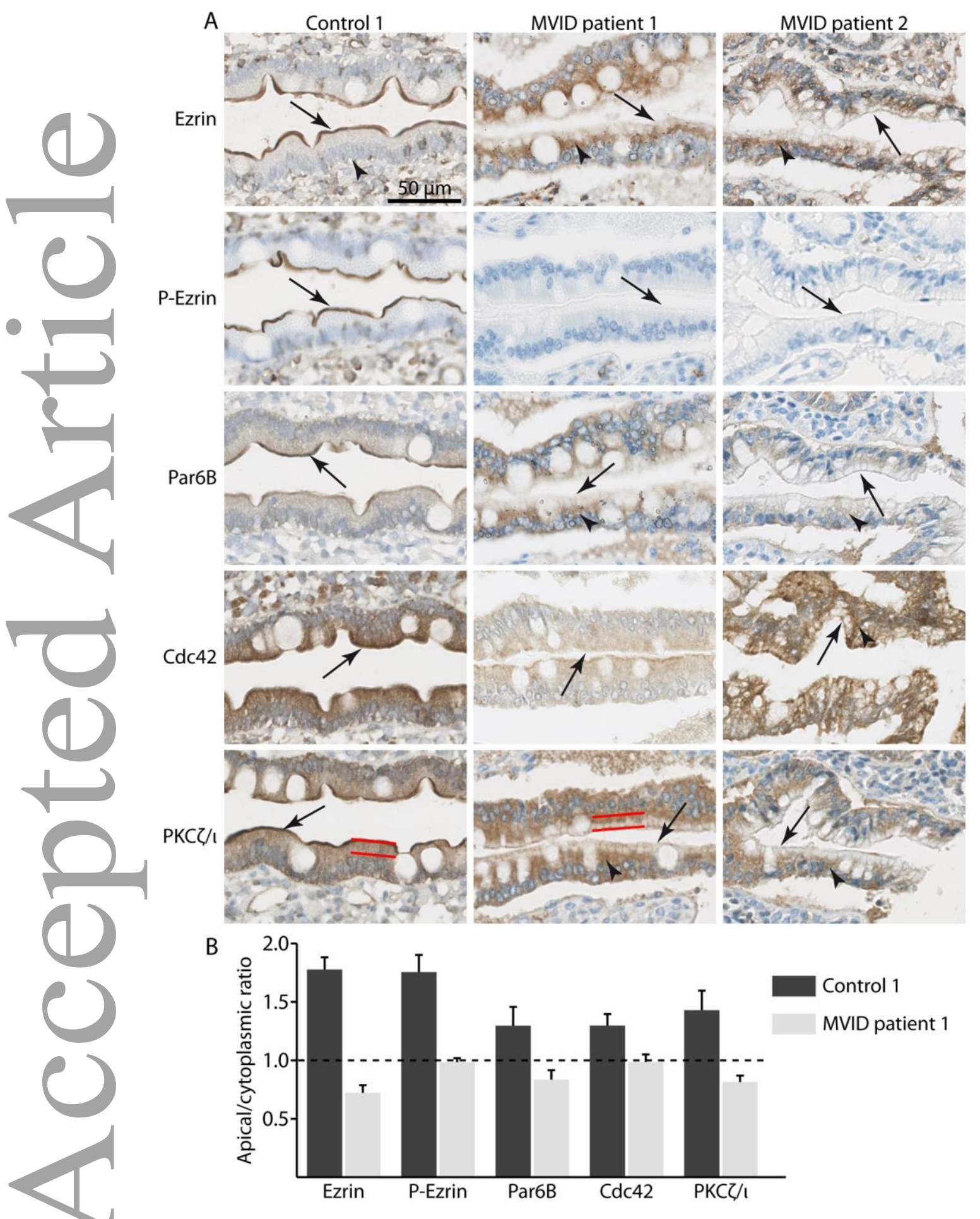

Figure 1

Michaux et al 
Figure 2: Localisation of polarity determinants and microvilli-like structures at the basal membrane in MVID patients

Ezrin, P-ezrin, Par6B, Cdc42 and PKCל/ı staining in control and MVID patient 3. In some villi of this patient apical markers are depleted from the apical membrane (arrows) but are found at the basal membrane (arrowheads) rather than intracellularly (middle panels); similar observations were made in patient 4; other markers showed the same pattern as P-Ezrin (not shown). Electron microscopy of patient 3 revealed less dense and disorganised microvilli at the apical membrane and microvilli-like structures (arrowheads) at the basal membrane (right panels); boxed areas are enlarged in the insets.

Scale bar is $50 \mu \mathrm{m}$ in the left and middle panels, $1 \mu \mathrm{m}$ in the right panels. 


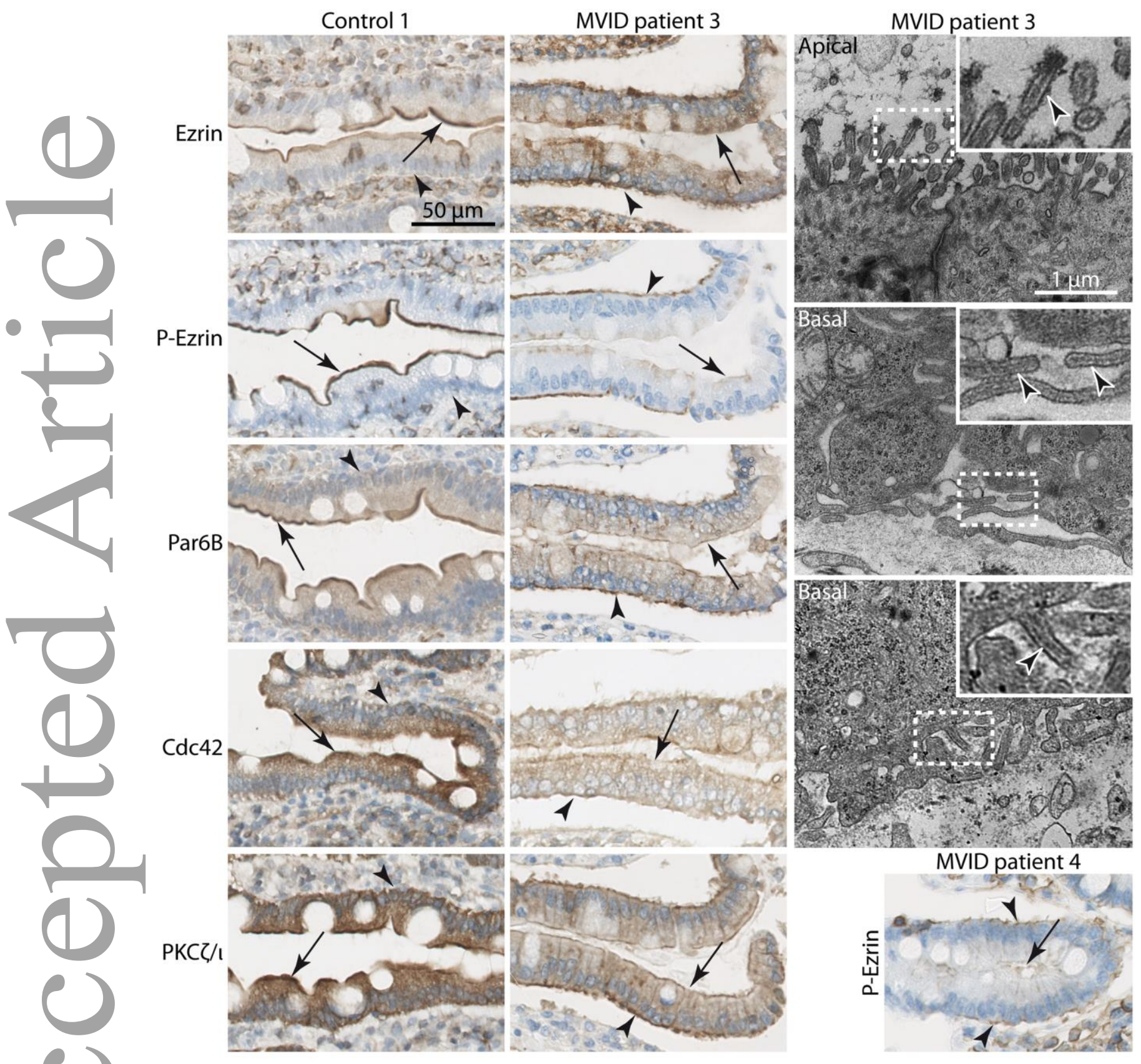

Figure 2

Michaux et al

Figure 3: Polarity inversion following MYO5B depletion in 3D grown Caco2 cells

A-D) Control and MYO5B (shMyo5B58) knock-down Caco2 cells plated as single-cell suspension on top Matrigel were fixed and stained after 10 days. Single confocal sections through the lumen of cysts stained for Ezrin (green; A), phospho-ezrin (green; B), Par6B (green; C), PKCל/ı (green; 
D), DPP IV (red; A-D), F actin (cyan; A-D), Dapi (blue in merged). All revealed markers (Ezrin, PEzrin, Par6B, PKC //, F actin, DPPIV) were concentrated at the apical surface (lumen indicated by an arrow) in control cysts while in MY05B knock-down cysts, Ezrin (A), P-Ezrin (B), Par6B (C) and PKC // (D) were accumulated in basal localisation (cyst limits indicated by an arrowhead). F actin and DPPIV were less frequently visible at the basal membrane (C). See also Fig S1 for shMyo5B76.

E) Quantification of phenotypes observed in control and Myo5B (shMyo5B58 and shMyo5B76) depleted cysts. The number of lumens and cyst polarity was quantified in three independent experiments ( $\mathrm{n}>100$ cysts/condition/experiment). About $60 \%$ of Myo5B depleted cysts displayed a single lumen and an inverted polarity. Error bars are S.E.M. 


\section{Figure 4: Localisation of polarity determinants in celiac disease and THE syndrome}

A) Ezrin, P-ezrin, Par6B, Cdc42 and PKC $/\llcorner$ staining in control (left panels), celiac disease (middle panels) and THE syndrome (right panels) enterocytes. All these markers are concentrated at the apical membrane (arrows) in control and affected enterocytes except Cdc42 which cannot be detected in THE syndrome patients (see also FigS2). The signal visible inside the epithelial layer in celiac disease patients is due to infiltrated lymphocytes.

B) Quantification of the apical/cytoplasmic ratio for each marker in control 2 and THE patient 1. The signal was measured along the apical membrane and in the cytoplasm as in Fig1. Measures were performed in ten different villi and the average ratio is shown. Error bars are S.E.M. Statistics: unpaired Student T-test. Only Cdc42 shows a significant difference. n.s.: not significant $(\mathrm{p}>0.05) ;{ }^{* * *} \mathrm{p}<1 \times 10^{-7}$. 


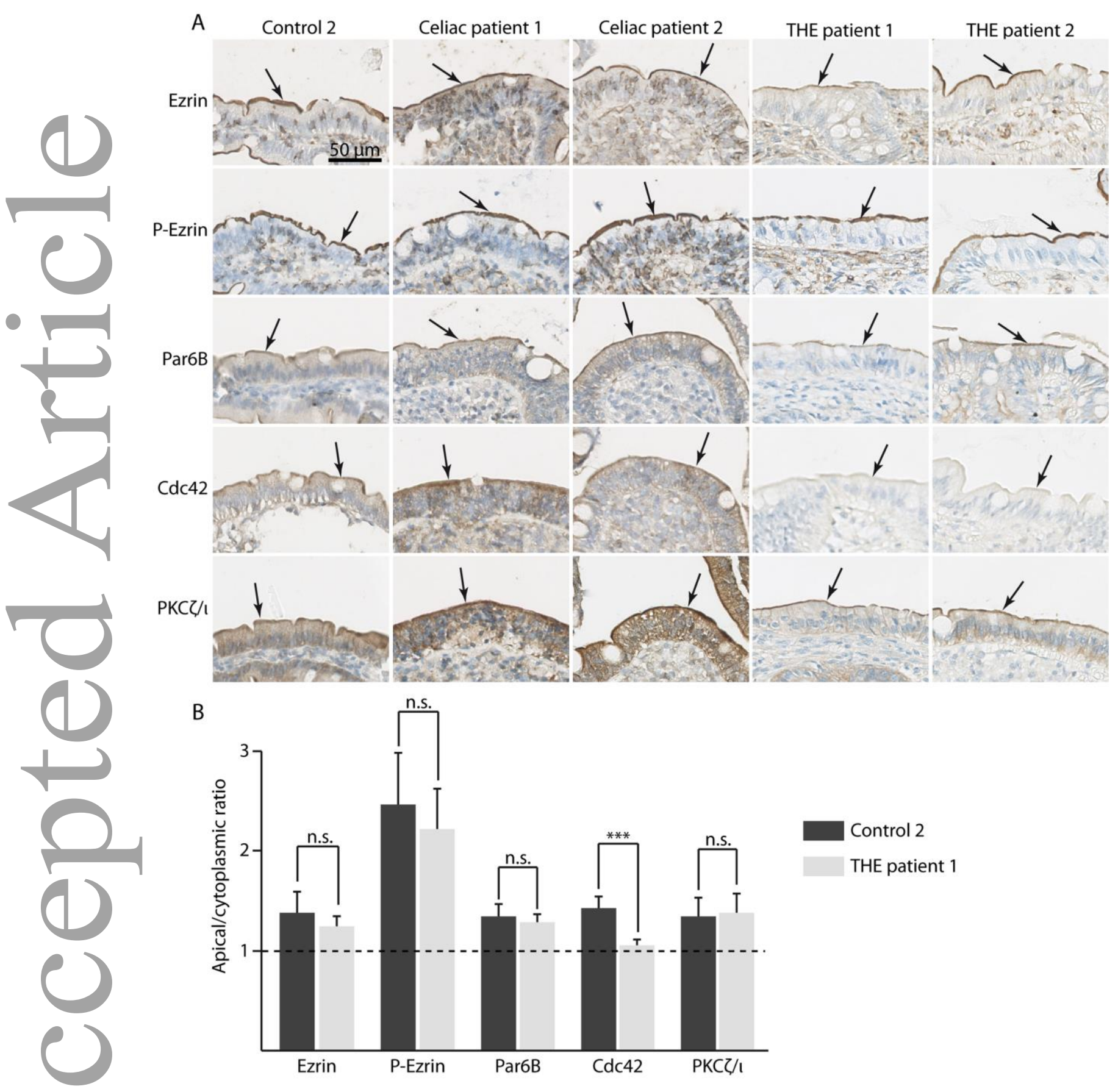

Figure 4

Michaux et al 


\section{SUPPLEMENTAL DATA}

\section{Figure S1: Myosin 5B depletion in Caco2 cells}

A) Cell extracts $(50 \mu \mathrm{g})$ from control and Myosin 5B knock down Caco2 cells were analyzed by SDS-PAGE and western blotting with antibodies against Myosin 5B or tubulin (Tub). Tubulin was used as an internal control for protein loading.

B) MY05B (shMyo5B76) knock-down Caco2 cells plated as single-cell suspension on top Matrigel were fixed and stained after 10 days. Single confocal sections through the lumen of cysts stained for Ezrin, phospho-Ezrin, Par6B, PKCろ/ı (green), DPP IV (red), F actin (cyan), Dapi (blue in merged). In MYO5B knock-down cysts, Ezrin, P-Ezrin, Par6B and PKCろ/ı were accumulated in basal localisation (cyst limits indicated by an arrowhead). See also Fig3.

\section{Figure S2: Localisation of Cdc42 in THE syndrome}

Cdc42 staining in THE syndrome and celiac disease; the apical membrane is shown by an arrow. The top row shows tissues from two THE syndrome and one celiac disease patients on a single slide to illustrate the efficiency of the 64533 Abcam anti-Cdc42 antibody. Boxed areas are enlarged in the second row. To confirm these observations we also stained all THE syndrome patients with the PA1-092 Thermo Fisher Pierce anti-Cdc42 antibody; the bottom row shows the comparative staining with the two anti-Cdc42 antibodies in one THE syndrome patient.

\section{Table S1: References of intestinal tissues}

MVID: Microvillus inclusion disease

SD/THE: Syndromic diarrhea / Tricho-hepato-enteric syndrome

The nature of the mutation is indicated when available. 


\section{GRAPHICAL ABSTRACT}

Myo5B is required for the correct localisation of the apical Par/Cdc42 module in intestinal cells. The loss of Myo5B in Microvillus Inclusion Disease (MVID) or in cultured cells induces the conversion of intracellular or basal membranes into apical-like membranes forming microvilli while the apical membrane presents a microvillus atrophy. The localisation of the apical Par/Cdc42 module is strictly correlated with the formation and maintenance of microvilli. 